\title{
Functional Molecular Switches of Mammalian G Protein-Coupled Bitter-Taste Receptors
}

Jérémie Topin ${ }^{+, a,}{ }^{,}$, Cédric Bouysset ${ }^{\dagger, \mathrm{a}}$, Jody Pacalon ${ }^{\mathrm{a}}$, Yiseul Kim ${ }^{\mathrm{b}}$, MeeRa Rhyu $^{\mathrm{b}}$, Sébastien Fiorucci ${ }^{\mathrm{a},{ }^{*}}$, Jérôme Golebiowski ${ }^{\mathrm{a}, \mathrm{c}}$

a Institut de Chimie de Nice UMR7272, Université Côte d'Azur, CNRS, France

${ }^{b}$ Korea Food Research Institute, 245 Iseo-myeon, Wanju-gun, Jeollabuk-do 55365, Republic of Korea

${ }^{\mathrm{c}}$ Department of Brain \& Cognitive Sciences, DGIST, 333, Techno JungAng, Daero, HyeongPoong Myeon, Daegu, 711-873, Republic of Korea

+ co-first authors

* jeremie.topin@univ-cotedazur.fr, sebastien.fiorucci@univ-cotedazur.fr

\section{Abstract}

Bitter taste receptors (TAS2Rs) are a poorly understood subgroup of G protein-coupled receptors (GPCRs). The experimental structure of these receptors has yet to be determined, and key-residues controlling their function remain mostly unknown. We designed an integrative approach to improve comparative modeling of TAS2Rs. Using current knowledge on class A GPCRs and existing experimental data in the literature as constraints, we pinpointed conserved motifs to entirely re-align the aminoacid sequences of TAS2Rs. We constructed accurate homology models of human TAS2Rs. As a test case, we examined the accuracy of the TAS2R16 model with site-directed mutagenesis and in vitro functional assays. This combination of in silico and in vitro results clarify sequence-function relationships and identify the functional molecular switches that encode agonist sensing and downstream signaling mechanisms within mammalian TAS2Rs sequences.

Classification: Biological sciences, Computational biology, and bioinformatics

Keywords: Bitter taste receptor, GPCR, structure-function relationships, integrative structural biology 


\section{Introduction}

Bitterness is one of the basic taste modalities detected by the gustatory system. It is generally considered to be a warning against the intake of noxious compounds ${ }^{1}$ and, as such, is often associated with disgust and food avoidance ${ }^{2}$. At the molecular level, this perception is initiated by the activation of bitter taste receptors. In humans, 25 genes functionally express these so-called type 2 taste receptors (TAS2Rs), which provide the capacity to detect a wide array of bitter chemicals ${ }^{3}$. Further, TAS2Rs are also ectopically expressed in non-chemosensory tissues, making them important emerging pharmacological targets ${ }^{4-6}$.

TAS2Rs are G protein-coupled receptors ${ }^{7}$ (GPCRs) classified as distantly related to class A GPCRs. They were previously classified with class F GPCRs ${ }^{8}$ and more recently as a separate sixth class evolved from class $A^{9,10}$. The sequence similarity between TAS2Rs and class A GPCRs is in the range of $14 \%-29 \%{ }^{11}$. Structure-based sequence alignment has placed TAS2Rs in the class A family, which contains the olfactory chemosensory receptors sub-family ${ }^{12}$. TAS2Rs have been recently labelled as class $T$ in the GPCR database (GPCRdb) (Fig. 1a) ${ }^{13}$.

Structurally, GPCRs are made up of seven transmembrane (TM) helices named TM1 to TM7 that form a bundle across the cell membrane. How GPCRs achieve specific robust signaling and how these functions are encoded in their sequences are pending fundamental questions. GPCR activation relies on so-called molecular switches, which allosterically connect the ligand binding pocket to the intracellular $G$ protein coupling site in order to trigger downstream signaling ${ }^{14}$. In class A GPCRs (including olfactory receptors, ORs), these molecular switches consist of conserved sequence motifs (Fig. 1c). The "toggle/transmission switch" CWXP ${ }^{\mathrm{TM} 6}$ (or FYGX ${ }^{\mathrm{TM} 6}$ in ORs) senses agonist binding. The other motifs, which propagate the signal, include the "hydrophobic connector" PIF ${ }^{\mathrm{TM} 3-5-6}$, the NPXXY ${ }^{\mathrm{TM} 7}$, the "ionic lock" DRY ${ }^{\mathrm{TM} 3}$, and a hydrophobic barrier between the last two ${ }^{15-18}$.

To date, experimental structures have not been determined for any TAS2Rs, but the following hallmark motifs have been defined based on sequence conservation: NGFI ${ }^{\mathrm{TM} 1}$, LAxSR ${ }^{\mathrm{TM} 2}, \mathrm{KIANFS}^{\mathrm{TM} 3}$, LLG ${ }^{\mathrm{TM} 4}$, $\mathrm{PF}^{\mathrm{TM} 5}, \mathrm{HXKALKT}^{\mathrm{TM} 6}, \mathrm{YFL}^{\mathrm{TM} 6}$, and PxxHSFIL ${ }^{\mathrm{TM} 7}$ 7. These conserved motifs are highly dissimilar between TAS2Rs and class A GPCRs (Fig. 1b,d and Table 1), leading to different sequence alignments. The main discrepancies occur in TM3, TM4, TM6, and TM7 $7^{11,19-30}$, making it difficult to infer TAS2R functional molecular switches. These discrepancies remain a central issue in understanding the complex allosteric TAS2R machinery. The present study aims to identify the molecular switches that control TAS2R functions. We present an integrative protocol that advances comparative modeling of TAS2Rs. Case studies of site-directed mutagenesis followed by in vitro functional assays on human TAS2R16 then evaluated the roles of the predicted molecular switches in TAS2Rs. 


\section{Methods}

\section{Sequence alignment}

Automatic multiple sequence alignment (MSA) of TAS2Rs was performed with class $A$ and class $F$ templates (labelled ClustalO and classF, respectively) using ClustalO ${ }^{31}$ with default settings in the Jalview interface $(\mathrm{v} 2.11 .0)^{32}$. These MSAs were not modified. Another MSA, labelled Chemosim, was completed using class A templates, 339 class II ORs and TAS2Rs. The Chemosim alignment was then manually refined using constraints from functional assays in the literature (as described in the results section). We specifically focused on the 339 class II ORs because they contain relevant motifs for TAS2Rs alignment and because TM sequence conservation is higher than in a mixture of class I and class II human ORs. TM segments were predicted by the PPM webserver ${ }^{33}$. The final Chemosim MSA is provided as an additional supporting file (TAS2R-OR-templates.pir).

\section{Template selection for comparative modeling of bitter taste receptors}

Class A GPCR templates were selected by submitting each of the 25 human TAS2Rs UniprotKB accession numbers to the Swiss-Model modeling server ${ }^{34}$. From the proposed templates for human TAS2Rs, 46 with at least $10 \%$ sequence identity were kept. Templates were then grouped by protein name and sorted by resolution and average sequence identity with TAS2Rs. The highest resolution template from each group was retained, resulting in 19 templates. Finally, six GPCR class A templates were selected to maximize structural diversity. As TAS2Rs have been suggested to be part of the same family as the frizzled receptors ${ }^{35}, 3$ class F GPCR templates were also considered: the human FZD4 receptor $^{36}$ and 2 structures of the human SMO receptor ${ }^{37}$. The PDB code for the six class A templates were as follows: rhodopsin (6FUF) $)^{38}, \beta 1$-adrenergic $(4 \mathrm{BVN})^{39}, \beta 2$-adrenergic receptor $(5 \mathrm{JQH})^{40}$, angiotensin II type 1 (4YAY $)^{41}$, chemokine receptor CXCR4 (3ODU $)^{42}$, serotonin receptor 5-HT2C $(6 B Q G)^{43}$.

\section{Integrative structural modeling of TAS2R}

Using the protocols described above (Chemosim, Gomodo, ClustalO, GPCRdb, BitterDB, and classF), we built a large number of 3D models and evaluated and ranked them using a meta-score defined as the average of the pocket and helicity score (Fig. 2). This score provides a unique descriptor that accounts for both GPCR structural requirements and TAS2R experimental constraints.

For each alignment (ClustalO, Chemosim, and classF) and each template, we generated 1000 homology models using Modeller v9.2144 with a maximum of 300 conjugate gradient minimization steps and refinement by molecular dynamics with simulated annealing ("md_level"=slow). The remaining 
parameters were set to default from the "automodel" class. The BitterDB and GPCRdb webservers provided additional 3D models of each TAS2R. The GOMoDo ${ }^{45}$ webserver was also used to automatically generate models of TAS2Rs based only on the sequence (labelled Gomodo in the analysis). Default options were used, excepting the number of models which was set to the maximum (99 models).

Evaluation of the model pocket score: To identify residues oriented toward the binding pocket, the following protocol was implemented in Python: i/ For each of the 25 human TAS2Rs, a reference 3D model was selected from the Chemosim models. All reference models were then structurally aligned to the TAS2R16 reference. ii/ A unique grid of points broadly covering the binding site of class A GPCRs was generated and aligned to the coordinates of the TAS2R16 reference. iii/ Each TAS2R model was aligned to its reference based on the alpha carbons of the TM residues. iv/ Residues whose sidechain center of mass (SCM) was within 8.0 angstroms of any grid point, and whose angle between the SCM, the alpha carbon, and any grid point was lower or equal to 30 degrees, were considered as oriented towards the pocket. Only residues annotated as involved in ligand binding were kept (see supporting file TAS2R-msa_annotated.xlsx). v/ The pocket score was calculated as the fraction of residues oriented towards the pocket for each TM, averaged across all TMs. 3D structure alignment was performed with MDAnalysis $v 1.0 .0^{46}$, and distance and angle calculations were performed with scipy $v 1.5 .0^{47}$ and numpy $v 1.19 .0^{48}$.

Evaluation of TM helicity: The Ramachandran number ${ }^{49}(R)$ was used to check the structural quality of the TM domains of each model produced. $R$, which is based on the $\varphi$ and $\psi$ dihedral angles, can be seen as a short numerical form of the Ramachandran plot. First, we analyzed the helicity of 358 class A GPCR X-ray structures to set the experimental range and found an average value of 0.35 . Thus, a residue was considered in an alpha-helix conformation if its $R$ value fell between 0.32 and 0.38 .

To discard misshapen 3D models having severe kinks in the middle of TM domains, we introduced a function based on $R$. We defined the function $f(r)=\operatorname{count}\left(\left|r_{i}-R_{r e f}\right| \leq \sigma\right)$, where $r$ is a moving subset of six consecutive $R$ values that are shifted forward until all $R$ values for a given TM helix have been sampled; $R_{\text {ref }}=0.35$ is the average $R$ value based on X-ray structures; and $\sigma=0.07$ is a parameter that was optimized to exclude misfolded TM proteins while keeping X-ray structures. If at any point the result of $f(r)$ was lower than 4 for any TM residue, the model was discarded. A helicity score $(\bar{H})$ was then calculated as the fraction of TM residues satisfying the condition: $\bar{H}=$ 
$\operatorname{count}\left(0.32 \leq R_{i} \leq 0.38\right) /$ length $(R)$. Among all considered X-ray structures, the minimum $\bar{H}$ value obtained was 0.789 . This threshold was used to filter out irrelevant models.

Assessing meta-score accuracy: The meta-score was defined as the average of the pocket and helicity scores. The relevance of the meta-score was assessed by building a homology model of the human smoothened receptor (class F) from a $\beta 2$-adrenoceptor template (class A, with a low shared sequence identity [9\%] with class F, PDB 5JQH). Using the experimental structure of a human smoothened receptor (PDB 4JKV), the RMSD of the best model was then calculated from the meta-score or from the scores available in Modeller or the QMEANBrane ${ }^{50}$ webserver. As shown in Fig. S2, the meta-score outperformed classical metrics when ranking GPCR models based on distantly related GPCR templates.

\section{Cell culture and transfection}

Plasmids encoding TAS2R16 and G16agust44 were constructed as previously described ${ }^{51}$. G16 ${ }_{\text {agust } 44}$ and TAS2R16 were cloned into a CMV promoter-based vector and expressed constitutively. Point mutations on the TAS2R16 clone were obtained from a commercial service (Macrogen Inc., Seoul, Republic of Korea), which also performed DNA sequencings of the mutant genes. The TAS2R16 and G16 agust44 expression plasmids were co-transfected (4:1) into HEK293T cells using Lipofectamine 2000 (Invitrogen, Carlsbad, CA, USA). Cellular responses were measured 18-24 h after transfection. Cells were cultured at $37^{\circ} \mathrm{C}$ in a humidified atmosphere of $5 \% \mathrm{CO}_{2}$. The culture medium was Dulbecco's modified Eagle's medium (DMEM) supplemented with $10 \%$ heat-inactivated fetal bovine serum (FBS), $100 \mathrm{IU} / \mathrm{ml}$ penicillin $\mathrm{G}, 100 \mu \mathrm{g} / \mathrm{ml}$ streptomycin, $2 \mathrm{mM}$ L-glutamine, and $1 \mathrm{mM}$ sodium pyruvate (Invitrogen).

\section{Quantitative measurement of intracellular $\mathrm{Ca}^{2+}$ in bitter taste receptors upon stimulation with salicin}

The compound-induced changes in cytosolic $\mathrm{Ca}^{2+}$ concentrations were measured using a FlexStation III microplate reader (Molecular Devices, Sunnyvale, CA, USA). Cells transfected with TAS2R16 were seeded onto 96-well black-wall CellBind surface plates (Corning, NY, USA). After 18-24 $\mathrm{h}$ seeding, the cells were washed with assay buffer $(130 \mathrm{mM} \mathrm{NaCl}, 10 \mathrm{mM}$ glucose, $5 \mathrm{mM} \mathrm{KCl}, 2 \mathrm{mM} \mathrm{CaCl}, 1.2 \mathrm{mM}$ $\mathrm{MgCl}_{2}$, and $100 \mathrm{mM} \mathrm{HEPES} ; \mathrm{pH} \mathrm{7.4)} \mathrm{and} \mathrm{incubated} \mathrm{in} \mathrm{the} \mathrm{dark,} \mathrm{first} \mathrm{at} 37^{\circ} \mathrm{C}$ for $30 \mathrm{~min}$, and then at $27^{\circ} \mathrm{C}$ for $15 \mathrm{~min}$ in assay buffer consisting of Calcium-4 (FLIPR Calcium 4 Assay Kit, Molecular Devices). After the samples were treated, the cell fluorescence intensity (excitation, $486 \mathrm{~nm}$; emission, $525 \mathrm{~nm}$ ) was measured. The results were plotted with $\Delta \mathrm{F} / \mathrm{F}^{0}$ on the $\mathrm{y}$-axis, where $\Delta \mathrm{F}$ is the change in Calcium- 4 fluorescence intensity at each time point, and $\mathrm{F}^{0}$ is the initial fluorescence intensity. The responses from at least three wells $(n=3)$ with the same stimulus were averaged. 


\section{Results and discussion}

\section{Matching conserved motifs between Class A GPCRs and TAS2Rs}

The prediction of TAS2Rs tertiary structure based on sequence similarity remains challenging due to discrepancy in the published alignment ${ }^{11,19-30}$. We have already shown that refining the sequence alignment of ORs with non-olfactory class A GPCRs by including site-directed mutagenesis produces relevant three-dimensional models of chemosensory receptors. These models have been supported by a large amount of experimental data ${ }^{16,18,52,53}$. We thus apply a similar integrative strategy to TAS2Rs. To overcome the lack of sequence similarity between TAS2Rs and GPCRs with known structures, we inserted 339 human class II OR sequences in the alignment. Subsequent manual data curation involved integration of site-directed mutagenesis data from the literature for 136 amino-acids positions, i.e. $45 \%$ of the entire TAS2Rs sequence (see ESI TAS2R-msa_annotated.xlsx). Our alignment (Fig. S1) highlights the key residues and consensus motifs in all human TAS2Rs, which correspond to the functional molecular switches in ORs and non-olfactory class A GPCRs (Fig. 1b,d). They are detailed above and summarized in Table 1.

TM1, 2 and 4 did not contain motifs involved in downstream signaling. In TM1, the NGFI ${ }^{\text {TM1-TAS2R }}$ motif

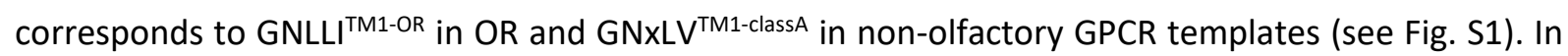
$T M 2, R^{2.50-T A S 2 R}$ in the LAxSR ${ }^{\text {TM2-TAS2R }}$ motif aligns with $D^{2.50-0 R / c l a s s A}$, which in class A GPCRs constitutes a sodium ion binding site that stabilizes inactive receptor conformations ${ }^{54}$. Position 2.50 in TAS2Rs is positively-charged and unlikely to be involved in sodium binding. Rather, it is hypothesized to stabilize the structure of TAS2Rs. ${ }^{21}$ The sequence alignment of TM4 was not straightforward, as it lacks the canonical $W^{4.50-O R / \text { classA }}$. The highly conserved leucine $L^{4.50}$ of the LLG $^{\text {TM4-TAS2R }}$ motif aligns with the most conserved W4.50-0R/class A.

TM3, 5, 6, and 7 contained functional molecular switches which have been identified in class A GPCR experimental structures ${ }^{14}$.

In TM3, $K^{3.50}$ in the KIANFS ${ }^{\text {TM3-TAS2R }}$ motif matches $R^{3.50}$ of the DRY ${ }^{\text {TM3-ClassA }}$ and MAYDRYVAIC ${ }^{\text {TM3-OR }}$ motifs. The DRY motif constitutes the ionic lock in ORs and non-olfactory class A GPCRs. This also aligns the highly conserved $\mathrm{L}^{3.43}$, with a leucine found at position 3.43 in both non olfactory class A GPCRs and OR (Table 1).

In TM5, the conserved $\mathrm{P}^{5.50}$ of the $\mathrm{PF}^{\mathrm{TM}}{ }^{\mathrm{TAS} 2 \mathrm{R}}$ motif corresponds to the PF $\mathrm{PF}^{\mathrm{TM} 5-\mathrm{OR}}$ and $\mathrm{P}^{\mathrm{TM} 5-\text {-lassA }}$ motifs/residue involved in the so-called "hydrophobic connector" ( $\left.P^{5.50}\right|^{3.40} \mathrm{~F}^{6.44}$ in class A GPCRs). Another conserved aromatic residue that is found in $52 \%$ of TAS2Rs, $F^{5.58}$, consistently aligns with the conserved $\mathrm{Y}^{5.58}$ known to be important for GPCR activation ${ }^{18,55}$. 
In TM6, the HXKALKT ${ }^{\text {TM6-TAS2R }}$ motif matches both a comparable motif in non-olfactory class A GPCRs and the typical OR motif RxKAFST ${ }^{\mathrm{TM} 6-O R}$. The "toggle/transmission switch" ( $\mathrm{CW}^{6.48} \mathrm{LP}^{\text {classA }}$ and $\mathrm{FY}^{6.48} \mathrm{G}^{\mathrm{OR}}$ ) aligns with the $\mathrm{YF}^{6.48} \mathrm{~L}$ motif in TAS2Rs. The location of this $\mathrm{YF}^{6.48} \mathrm{~L}$ motif at the bottom of the pocket is consistent with site-directed mutagenesis results, suggesting a ligand-sensing role, as is the case for class A GPCRs ${ }^{16,56}$.

The extracellular part of TM7 is well-documented to belong to the ligand binding pocket in TAS2Rs and other GPCRs ${ }^{20,24,56}$. This is consistent with its high sequence variability (see Fig. S1). TM7 intracellular residues show higher conservation, as they are involved in GPCR signaling ${ }^{16,56}$. These conserved motifs, however, show little similarity between TAS2Rs and other GPCRs. Here, the comparison with ORs is highly instructive: from the $P^{7.46} X L N P^{7.50} \times I Y^{T M 7-O R}$ motif found in ORs, $P^{7.46}$ is shared with TAS2Rs, and $N P^{7.50} \mathrm{xxY}$ is found in other class A GPCRs. $\mathrm{P}^{7.46}$ and $\mathrm{P}^{7.50}$ are conserved in $76 \%$ and $28 \%$ of human TAS2Rs, respectively. The PXXHSFIL ${ }^{\text {TM7-TAS2R }}$ motif is consequently aligned with PXLNPXIYTM7-OR, which itself matches the highly conserved $x x x N P x x Y^{T M 7-\text { class }^{2}}$ motif $^{20}$.

\section{Predicted tertiary structure of TAS2Rs}

Based on this refined alignment, we tested various protocols and structural templates to build accurate 3D homology models of TAS2Rs. Among the TAS2Rs, receptors TAS2R14, 16, and 46 were selected to evaluate the approach, as previous work on these receptors involving site-directed mutagenesis provides data to determine the residues within their binding pocket. According to our meta-score, the best models of these three receptors were obtained using the Chemosim approach and a single template, either the $\beta 2$-adrenoceptor (PDB 5JQH) or the $\beta 1$-adrenoceptor (PDB 4BVN) structure (Fig. 2 \& S3). The performance of each protocol is compared in Fig. S3 and S4. Gomodo and ClustalO approaches led to comparable models, with slight improvement over BitterDB and, in most cases, substantial improvement over GPCRdb. The use of class F templates systematically led to models with misfolded helices (Fig. S4).

These models and analysis were then extrapolated to the full human TAS2Rs repertoire. Even if limited experimental data is available, we were able to define a consensus TAS2R cavity based on the positions identified simultaneously in TAS2R14, 16 and 46. We also extended the definition of a specific TAS2R cavity to residues identified by site-directed mutagenesis. The best models for the entire TAS2R family were obtained using GPCR templates in their closed conformation (Fig. S6), with the exception of TAS2R38, for which the open-conformation $5-\mathrm{HT}_{2}$ receptor (PDB 6BQG) was best. On average, the templates 5JQH, 4BVN, and 2RH1, all of which correspond to adrenergic receptors, performed best. In this study, we found no relationship between the performance of the protocols and the percentage 
sequence identity of the templates used to build the models. At $10-15 \%$, the sequence identity between TAS2Rs and class A templates is too low to be a discriminating criterion.

The best Chemosim model obtained for each human TAS2R is provided as a PDB file in the supporting information. Projecting TAS2Rs sequence conservation onto the 3D structure showed that the models retain the structural characteristics of the GPCR (Fig. S5). The most conserved residues were located in the intracellular region of the receptor that binds the G protein, while the greatest variability was found in the extracellular ligand-binding pocket. Analysis of the binding cavity (Fig. S7) revealed high diversity within the hTAS2Rs family. The pocket volume ranged up to $400 \AA^{3}$ and $700 \AA^{3}$ for hTAS2R13 and hTAS2R39, respectively, corresponding to the structural features of a GPCR ${ }^{57}$. Although no obvious structure-function relationship was revealed by the analysis of the cavity volume, the hydrophobicity partially correlated with the receptor range of response. The binding cavities of TAS2Rs with broad ligand spectrums tended to be more hydrophobic than those of narrow-spectrum receptors (Fig. S7), consistent with previous studies showing a correlation between hydrophobicity and GPCR promiscuity ${ }^{58,59}$.

\section{Evaluating the function role of molecular switches}

To evaluate the functional role of the predicted molecular switches, twelve residue positions on TAS2R16 were subjected to site-directed mutagenesis followed by in vitro functional assays with salicin (Fig. 3 and Table S2). The residues mostly belonged to TM3 and TM6, which, in GPCRs, are well-known to be involved in agonist sensing and activation ${ }^{14}$.

Using our model as a basis, we investigated residues found in the ligand binding pocket $\left(90^{3.35}, 91^{3.36}\right.$, and $\left.185^{5.47}\right)$ and at or around the predicted molecular switches $\left(45^{2.39}, 97^{3.41}, 221^{6.29}, 222^{6.30}, 236^{6.44}\right.$, and $239^{6.47}$ ). Residues $42^{\mathrm{ICL} 1}, 43^{\mathrm{ICL} 1}$, and $100^{3.44}$ were predicted to be far from the molecular switches. All mutants showed a specific, dose-dependent response to salicin (Fig. 3), confirming that they are expressed and functional at the cell surface.

The $\mathrm{L} 42^{\mathrm{ILL1}} \mathrm{A} / \mathrm{S}, \mathrm{M} 43^{\mathrm{ICL1}} \mathrm{A}$, and $\mathrm{T} 100^{3.44} \mathrm{~A}$ mutations served as negative controls (Table $\mathrm{S} 2$ ) and generally did not statistically affect salicin potency (Fig. 3 and Table S3). Only mutation of position 43 to a serine induced a weak decrease of salicin-dependent response in TAS2R16 compared to WT.

The TASR216 $190 \mathrm{~A} / \mathrm{S}^{3.35}, \mathrm{~L} \mathrm{~L} 1 \mathrm{~A} / \mathrm{S}^{3.36}$, and $\mathrm{L} 185 \mathrm{H}^{5.47}$ mutants showed a reduced response to salicin, consistent with their orientation toward the interior of the receptor bundle (Fig. 3 and Table S3). Positions 3.35 and 5.47 have been previously reported to directly interact with ligands ${ }^{26,30,60}$. Position $239^{6.47}$ is conserved as $Y(64 \%)$ and $F(8 \%)$ in human TAS2Rs (Fig. 4a). In mammals, an aromatic residue $(\mathrm{F}, \mathrm{Y}$ or $\mathrm{H})$ is also found in $85 \%$ of the sequences. Conservation of an aromatic residue also occurs in $\mathrm{ORs}^{16}$. The $\mathrm{Y}^{2} 39 \mathrm{~F}^{6.47}$ mutation decreased the potency of salicin by a factor of 11 , confirming 
its importance in receptor activation (Fig. 3). Position Y2396.47 corresponded to Y239 and Y241 in TAS2R10 and TAS2R46, respectively. For both of these receptors, the tyrosine to phenylalanine mutation led to a significant reduction in ligand responsiveness. Further, we found that the introduction of an alanine at this position eliminated any response to salicin (data not shown). Born et al. also observed a complete loss of response to agonists with the Y239A ${ }^{6.47}$ TAS2R10 construction $^{61}$. Altogether, these observations highlight the functionnal equivalence of the $\mathrm{Y}^{6.47} \mathrm{FLx}$ motif in TAS2Rs with the $F^{6.47} \mathrm{YGX}$ in $\mathrm{ORs}^{16}$ and the $\mathrm{C}^{6.47} \mathrm{WLP}^{14}$ in non-olfactory class A GPCRs ${ }^{9}$. This motif is particularly important as it forms part of the cradle of the binding pocket and senses the presence of agonists ${ }^{56}$. Adjacent to $\mathrm{Y} 239^{6.47}$, the aromatic residue $\mathrm{F} 240^{6.48}$ is conserved as aromatic in $72 \%$ of human TAS2Rs and in $67 \%$ of mammalian TAS2Rs. As the toggle-switch residue, its nature and function in agonist sensing is similar in ORs (conserved as $\left.\mathrm{F}^{6.48}\right)^{16}$ and non-olfactory GPCRs (conserved as $\mathrm{W}^{6.48}$ ) $^{14}$. F240 $60^{6.48}$ has previously been reported to affect TAS2R16 agonist response. Sakurai et al. showed that mutation of $F 240^{6.48}$ to a leucine residue in TAS2R16 drastically alters the function of the receptor, while mutation to aromatic residues ( $\mathrm{Y}$ and $\mathrm{W}$ ) leads to moderate changes in the EC50 $0^{19}$. Further, the potencies of various other agonists were affected in the same manner, highlighting the critical role this residue plays in signal initiation, as is the case for numerous class A GPCRs ${ }^{14-16}$.

The hydrophobic connector molecular switch involved in class A GPCRs activation ${ }^{15}$ was conserved as $\mathrm{P}^{5.50} \mathrm{I}^{3.40} \mathrm{~F}^{6.44} 14,15,17$. Similarly to other TAS2Rs, a $\mathrm{P}^{5.50} \mathrm{~A}^{3.40} \mathrm{~F}^{6.44}$ motif (Fig. 4b) was located at the core of TAS2R16, close to the cradle of the binding pocket. In class A GPCRs, this motif, together with NPXxY $Y^{\mathrm{TM} 7}$, holds a central role in receptor signaling, ligand-independent constitutive activation, and $\beta$-arrestin signaling in the $\beta 2$-adrenoceptor ${ }^{17}$. It is plausible that this motif has similar functions in TAS2Rs ${ }^{62}$, as suggested by the modulated response to salicin we found in our mutants (Fig. 3). F236 6.44 , conserved in $75 \%$ of mammalian TAS2Rs as $\mathrm{Y} / \mathrm{F}$ (Fig. 4b), is predicted to be part of the hydrophobic connector molecular switch. The F236A ${ }^{6.44}$ TAS2R16 mutant consistently showed a significantly weaker response to salicin, while no difference in response was found for the $F 236 \mathrm{Q}^{6.44}$ mutant. In a previous study, Thomas et al. found that a F236Y $\mathrm{Y}^{6.44}$ mutation prevented agonist-dependent signaling ${ }^{25}$. In TAS2R14, an alanine residue occupies position 6.44 , and mutation to a leucine leads to a decrease in receptor sensitivity to numerous ligands ${ }^{55}$.

Adjacent to position $3.40,597^{3.41}$ does not belong to the binding pocket and points toward the membrane. In accordance with a previous report showing its importance for TAS2R16 trafficking ${ }^{26}$, the S97A $\mathrm{A}^{3.41}$ mutation altered receptor response (gain of function).

Our model predicted that $\mathrm{V} 45^{2.39}$ is part of a hydrophobic cluster in the intracellular part of TM2 and is conserved as a hydrophobic residue in $72 \%$ of TAS2Rs. This hydrophobic area occurs near the highly

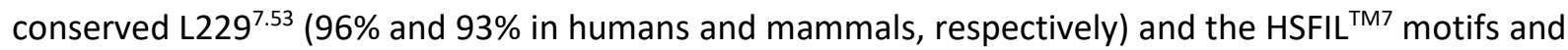
likely forms part of the hydrophobic barrier that prevents flooding of the intracellular region. Mutating 
V45 2.39 into a hydrophilic residue (S) strongly altered salicin activation both in this work and in the literature ${ }^{26}$; substitution with a bulkier hydrophobic residue $(F)$ was better tolerated.

In TM6, position 6.29 and adjacent residues have been documented to control G protein selectivity in class A GPCRs ${ }^{63}$. A22 $1^{6.29}$ and $\mathrm{H} 222^{6.30}$ are conserved in $60 \%$ and $92 \%$ of human TAS2Rs, respectively, and in $70 \%$ and $94 \%$ of mammalian TAS2Rs (Fig. 4c). Position $222^{6.30}$ is an arginine in TAS2R16. Salicin induced reduced responses in the $\mathrm{A} 221 \mathrm{~L}^{6.29}$ and $\mathrm{R} 222 \mathrm{~A}^{6.30}$ mutants, whereas the response of the $\mathrm{R} 222 \mathrm{H}^{6.30}$ mutant was not statistically different from the WT. In TASR2R4, the H233 $\mathrm{A}^{6.30}$ mutation inhibited the response to quinine ${ }^{64}$. Altogether, these findings highlight the need for a positive charge at position 6.30 for $\mathrm{G}$ protein-coupling and selectivity.

\section{Conclusions}

This study elucidates key residues and consensus functional motifs of bitter taste receptors (TAS2Rs) using a combination of bioinformatics, molecular modeling, and in vitro assays. The consensus sequence motifs match well-known ones in class A GPCRs. Further, we performed sequence alignment of human TAS2Rs with olfactory and non-olfactory class A GPCRs, including residue conservation and experimental data as constraints. Using site-directed mutagenesis, we then evaluated the functional roles of these motifs in TAS2R16 as a case study. In addition to the residues lining the binding pocket, we identified the "toggle/transmission switch" (the $\mathrm{YF}^{6.48} \mathrm{~L}$ motif in TM6) and the "hydrophobic connector" $\left(\mathrm{P}^{5.50} \mathrm{~A}^{3.40} \mathrm{~F}^{6.44}\right)$ for agonist sensing. Other molecular switches were identified in the intracellular regions of TM6 and TM7 that are suggested to be involved in G protein selectivity or in receptor activation. These molecular switches extends to mammalian TAS2Rs (see supporting files). The approach, templates, and 3D model provided in this study serve as a foundation for rational design of specific TAS2Rs agonists and antagonists and for decoding sequence-structure-function relationships in these receptors.

\section{Code and data availability}

The scripts used to generate and analyze the models as well as PDB files of TAS2Rs 3D models with the highest meta-score have been deposited on GitHub. (https://github.com/chemosim-lab/TAS2R_data) 
bioRxiv preprint doi: https://doi.org/10.1101/2020.10.23.348706; this version posted June 21,2021 . The copyright holder for this preprint

(which was not certified by peer review) is the author/funder, who has granted bioRxiv a license to display the preprint in perpetuity. It is made available under aCC-BY-NC-ND 4.0 International license.

\section{Author contributions}

$\mathrm{JT}^{\dagger}, \mathrm{CB}^{\dagger}$, and JP performed numerical modeling. $\mathrm{YK}$ and $\mathrm{MR}$ conducted functional assays. JT, $\mathrm{CB}$, and $\mathrm{SF}$ performed data curation. JT, CB, JP, YK, and MR conducted formal analyses. JT, SF, and JG supervised and managed the study and wrote the paper. MR and JG provided resources for this study.

\section{Conflicts of interest}

The authors declare no competing financial interest.

\section{Acknowledgements}

The authors thank Dr. Xiaojing Cong for fruitful discussion and critical review of the manuscript. This work was funded by the French Ministry of Higher Education and Research [PhD Fellowship to CB], by the National Research Foundation of Korea (NRF) [grant number NRF2020R1A2C2004661], by GIRACT (Geneva, Switzerland) [9th European PhD in Flavor Research Bursaries for first year students to CB], and the Gen Foundation (Registered UK Charity No. 1071026), a charitable trust that primarily funds research in natural sciences, particularly food sciences/technology [grant to CB and JT]. We also benefited from funding by the French government through the UCAJEDI "Investments in the Future" project, managed by the ANR [grant No. ANR-15-IDEX-01 to SF and JG]. Computation for the work described in this paper was supported by the Universite Côte d'Azur's Center for High-Performance Computing. 
a

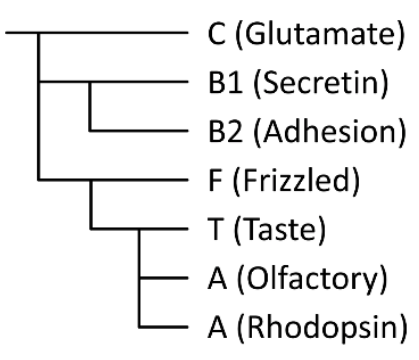

C

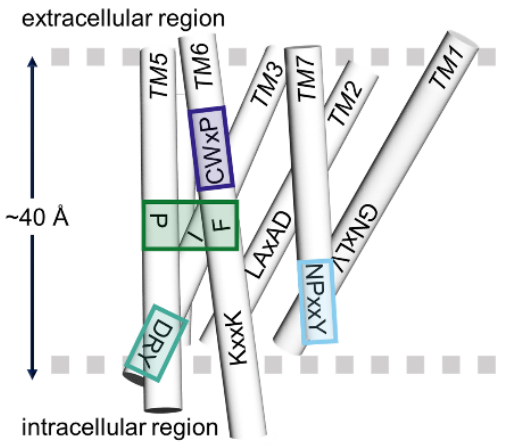

b

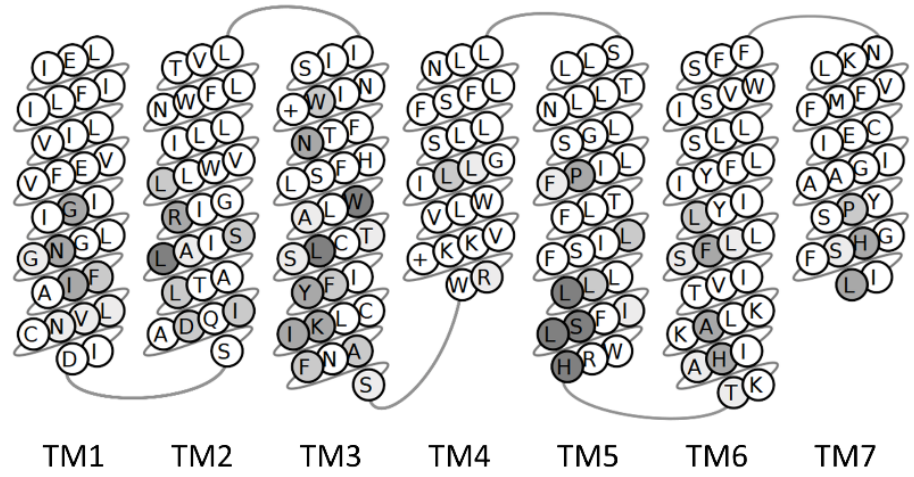

d

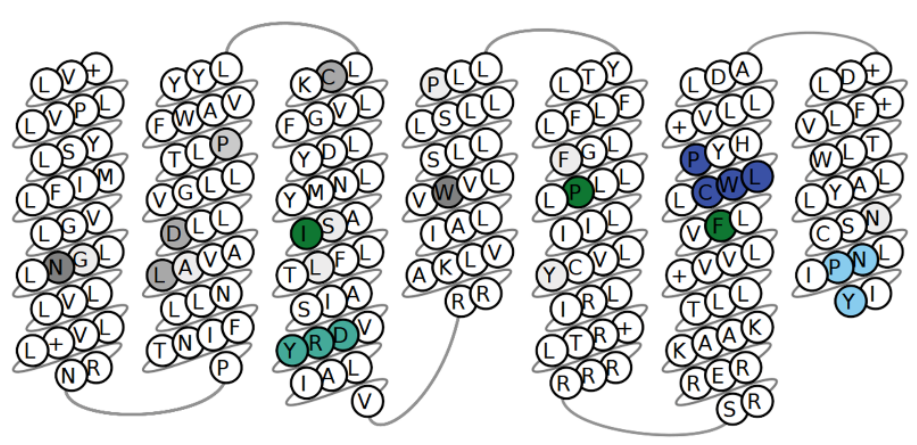

Figure 1. a) Schematic phylogenetic tree of GPCR classes according to Cvicek et al. ${ }^{12}$. b) Snake plot representation of transmembrane segments (TM) of mammalian TAS2Rs consensus sequences, colored in grey scale according to sequence conservation. c) Non-olfactory class A GPCR sequence hallmarks (transmission switch in blue, hydrophobic connector in green, ionic lock in sea green, hydrophobic barrier in light blue). d) Snake plot representation of non-olfactory class A GPCR consensus sequences. 
a Integrative strategy for TAS2R binding cavity identification
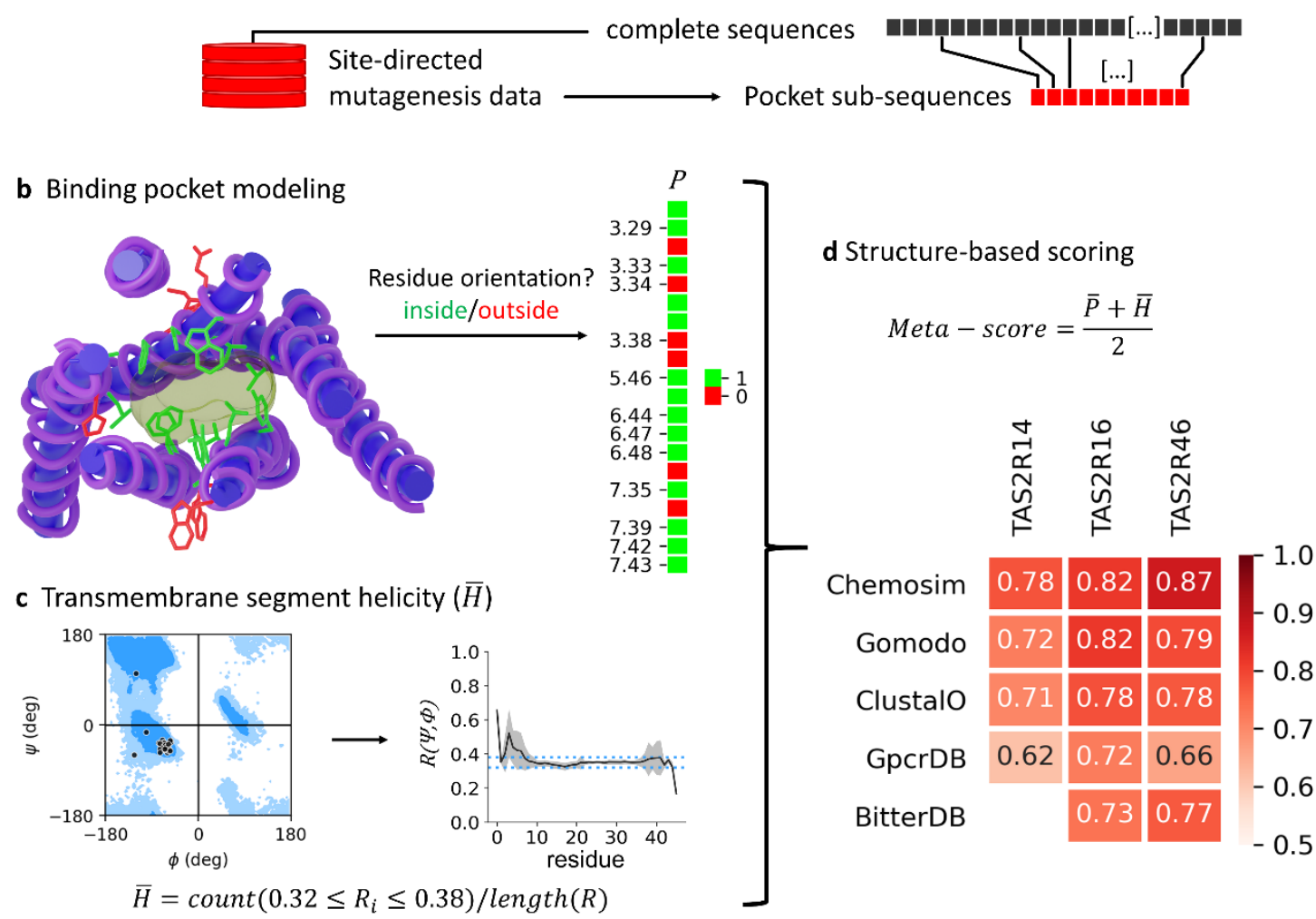

Figure 2. a) An integrative approach to identify the TAS2R binding pocket that is used as a constraint in comparative modeling with the Chemosim protocol. b) A pocket fingerprint was extracted based on the positions of binding residues in the 3D model. The light brown surface represents the binding pocket. c) The helicity of the TM segment was analyzed and d) combined with the pocket fingerprint to calculate a structure-based normalized meta-score. The meta-scores of the best 3D models of TAS2R14, 16 and 46 structures generated by the different comparative modeling protocols are shown in panel d). 
a
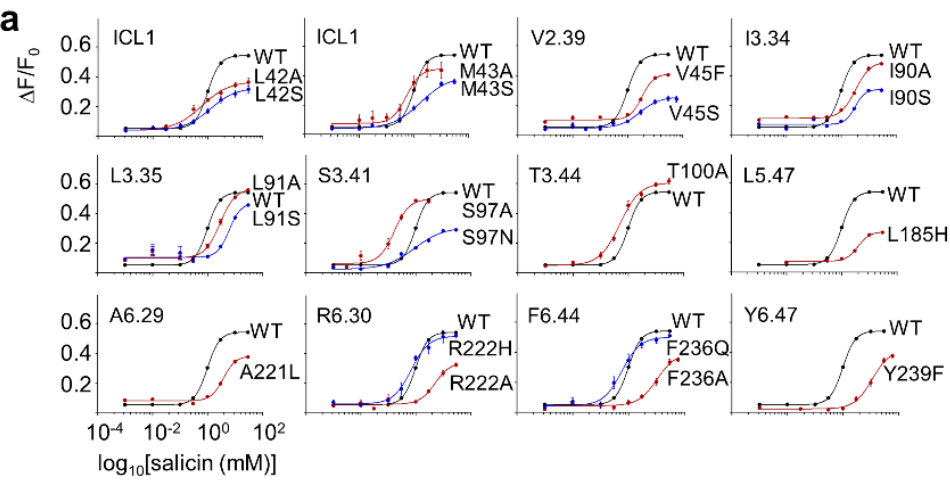

b

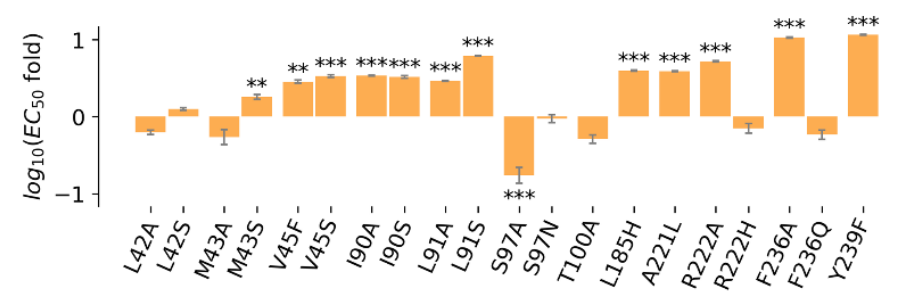

C

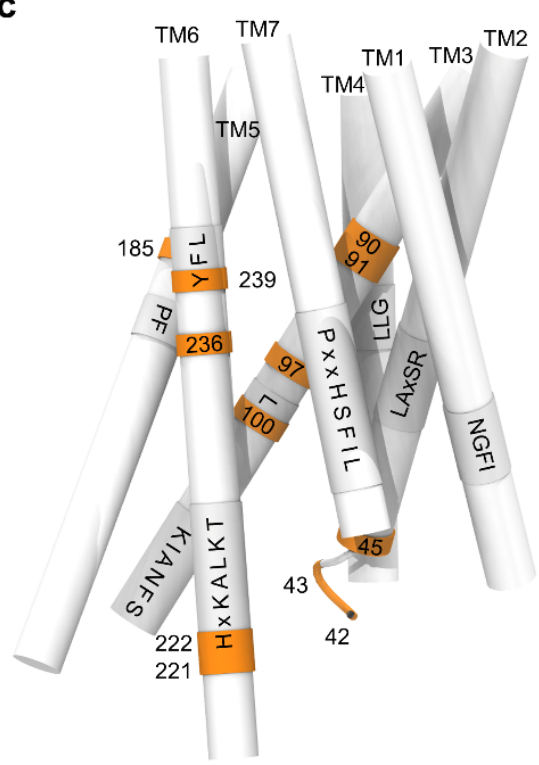

Figure 3. a) In vitro functional assays of wild-type (WT) TAS2R16 and single-point mutants stimulated by salicin. b) $\mathrm{EC}_{50}$ fold (compared to WT) expressed as $\log \left(\mathrm{EC}_{50}(\mathrm{MUT}) / \mathrm{EC}_{50}(\mathrm{WT})\right.$ ) for the twenty TAS2R16 mutants considered in this study. Positive values indicate a reduced response to salicin in the mutated receptor compared to the WT. ${ }^{* * *} p<0.001,{ }^{* *} p<0.01$, and ${ }^{*} p<0.05$ versus the WT group (one-way ANOVA followed by Dunnett's test). c) Representative structure of TAS2R16 highlighting the location of the mutated residues. The TM domains are presented as sticks. The positions of mutated residues are colored in orange, and the molecular switches revealed by the sequence alignment are indicated on the structure. 


\section{Class A GPCR \\ ( $\beta 2$-adrenoceptor)}

a Transmission switch

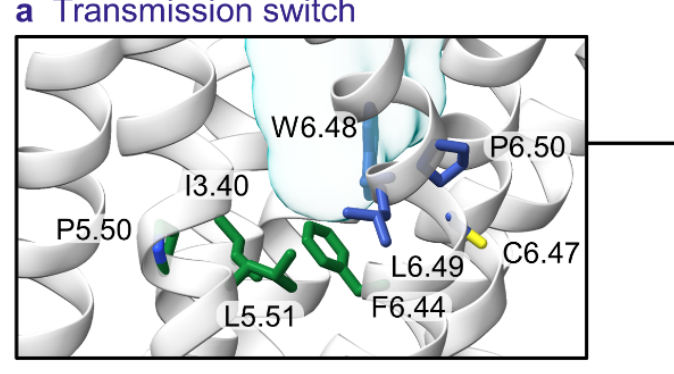

Conserved motifs

$\hat{x} \dot{2} \hat{\infty}$
TAS2R

(TAS2R46 model)

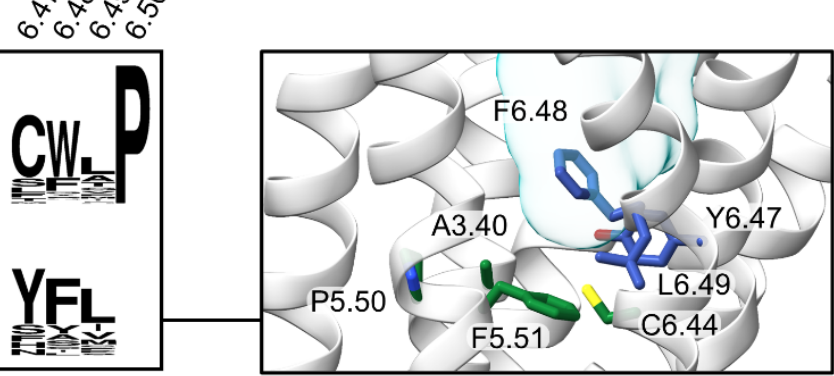

b Hydrophobic connector
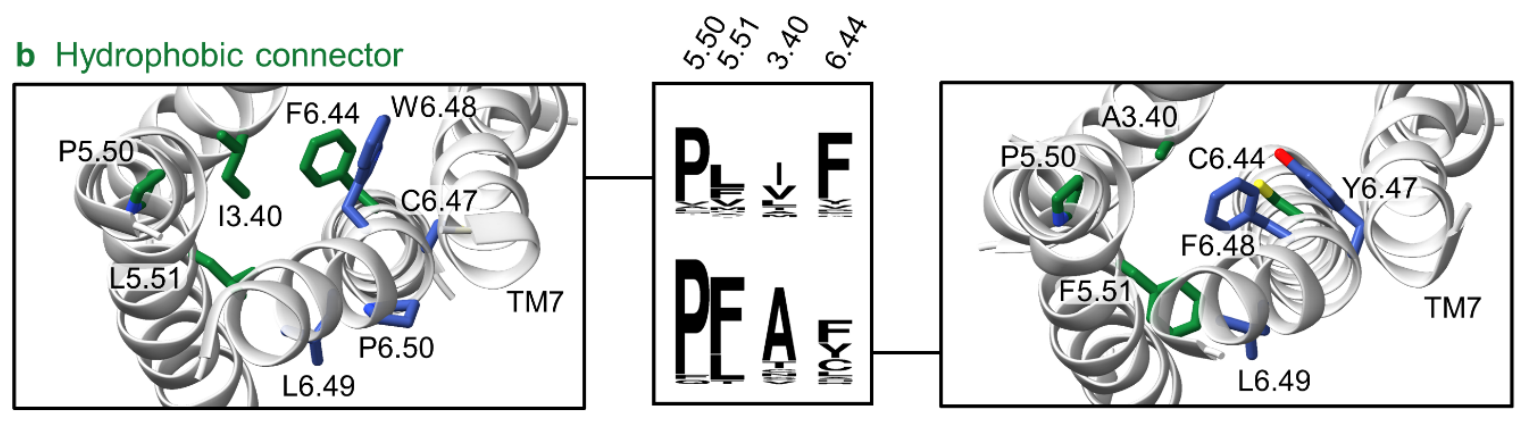

c G protein coupling
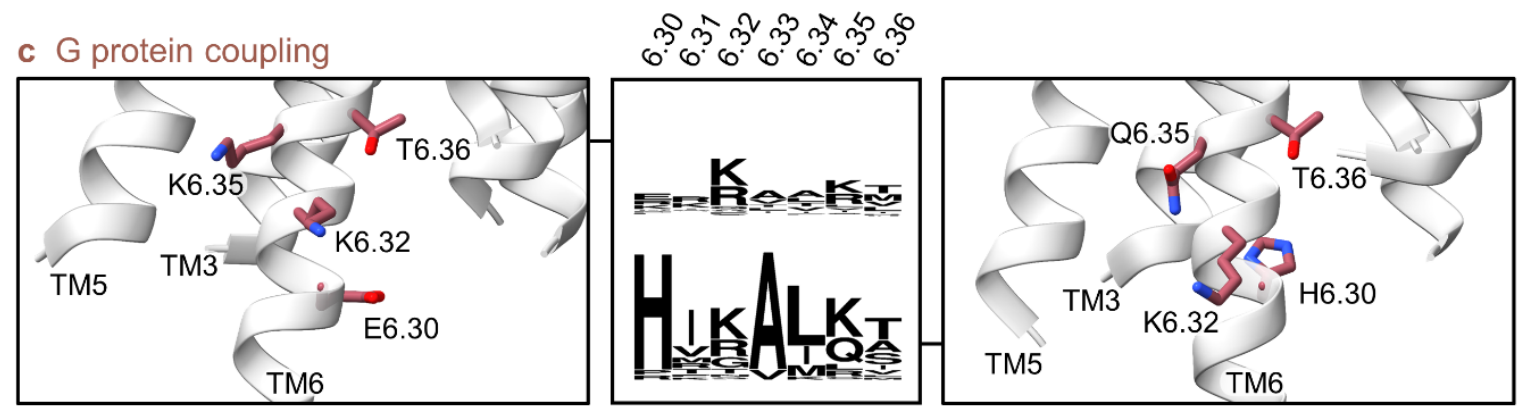

Figure 4. Sequence logos and molecular details of conserved motifs involved in the activation mechanism of class A GPCRs and TAS2Rs, i.e. a) the transmission switch (colored in blue), b) the hydrophobic connector (in green), and c) the G protein-coupling region (in red). The binding pocket is depicted as a pale blue surface. The structure of the $\beta 2$-adrenoceptor is taken from PDB code 5JQH. 
bioRxiv preprint doi: https://doi.orq/10.1101/2020.10.23.348706; this version posted June 21,2021 . The copyriaht holder for this preprint (which was not certified by peer review) is the author/funder, who has granted bioRxiv a license to display the preprint in perpetuity. It is made available under aCC-BY-NC-ND 4.0 International license.

Table 1. Key residues and consensus motifs.

Superscripts refer to the Ballesteros-Weinstein numbering scheme.

\begin{tabular}{|c|c|c|c|}
\hline TM & class A GPCR & OR & TAS2R \\
\hline 1 & $G N^{1.50} x L V$ & $\mathrm{GN}^{1.50} \mathrm{LLI}$ & $\mathrm{N}^{1.50} \mathrm{GFI}$ \\
\hline 2 & $\mathrm{LAxAD}^{2.50}$ & LSFxD $^{2.50}$ & $\operatorname{LAxSR}^{2.50}$ \\
\hline \multirow{2}{*}{3} & $\mathrm{~L}^{3.43}$ & $\mathrm{~L}^{3.43}$ & $\mathrm{~L}^{3.43}$ \\
\hline & $\mathrm{DR}^{3.50 \mathrm{Y}}$ & MAYDR ${ }^{3.50}$ YVAIC & $\mathrm{K}^{3.50} \mathrm{IANFS}$ \\
\hline 4 & $W^{4.50}$ & $W^{4.50}$ & $\mathrm{~L}^{4.50} \mathrm{LG}$ \\
\hline \multirow{2}{*}{5} & $\mathrm{P}^{5.50}$ & $\mathrm{P}^{5.50} \mathrm{~F}$ & $\mathrm{P}^{5.50} \mathrm{~F}$ \\
\hline & $\gamma^{5.58}$ & $Y^{5.58}$ & $F^{5.58}$ \\
\hline \multirow{2}{*}{6} & $\overline{K^{6.32} \mathrm{xxK}}$ & $\mathrm{RxK}^{6.32} \mathrm{AFSTC}$ & $\mathrm{HxK}^{6.32} \mathrm{ALKT}$ \\
\hline & $\mathrm{CW}^{6.48} \mathrm{LP}$ & $\mathrm{FY} Y^{6.48} \mathrm{G}$ & $Y F^{6.48} \mathrm{~L}$ \\
\hline 7 & SxxNP $7.50 x x Y$ & PXXNP $7.50 x I Y$ & $\mathrm{PxxHS} S^{7.50} \mathrm{FIL}$ \\
\hline
\end{tabular}




\section{References}

$1 \quad$ Lindemann, B. Receptors and transduction in taste. Nature 413, 219-225 (2001).

2 Meyerhof, W. in Reviews of physiology, biochemistry and pharmacology 37-72 (Springer, 2005).

3 Mueller, K. L. et al. The receptors and coding logic for bitter taste. Nature 434, 225-229 (2005).

4 Lee, S.-J., Depoortere, I. \& Hatt, H. Therapeutic potential of ectopic olfactory and taste receptors. Nature Reviews Drug Discovery 18, 116-138 (2019).

5 Foster, S. et al. Novel bitter taste receptor ligands elicit $G$ protein-dependent negative inotropic effects in mouse heart (LB572). The FASEB Journal 28, LB572 (2014).

6 Malki, A. et al. Class I odorant receptors, TAS1R and TAS2R taste receptors, are markers for subpopulations of circulating leukocytes. Journal of leukocyte biology 97, 533-545 (2015).

7 Adler, E. et al. A novel family of mammalian taste receptors. Cell 100, 693-702 (2000).

8 Fredriksson, R., Lagerström, M. C., Lundin, L.-G. \& Schiöth, H. B. The G-protein-coupled receptors in the human genome form five main families. Phylogenetic analysis, paralogon groups, and fingerprints. Molecular pharmacology 63, 1256-1272 (2003).

9 Nordström, K. J., Sällman Almén, M., Edstam, M. M., Fredriksson, R. \& Schiöth, H. B. Independent HHsearch, Needleman-Wunsch-based, and motif analyses reveal the overall hierarchy for most of the $\mathrm{G}$ protein-coupled receptor families. Molecular biology and evolution 28, 2471-2480 (2011).

10 Krishnan, A., Almén, M. S., Fredriksson, R. \& Schiöth, H. B. The origin of GPCRs: identification of mammalian like Rhodopsin, Adhesion, Glutamate and Frizzled GPCRs in fungi. PloS one 7 , e29817 (2012).

11 Di Pizio, A. et al. in Methods in cell biology Vol. 132 401-427 (Elsevier, 2016).

12 Cvicek, V., Goddard III, W. A. \& Abrol, R. Structure-based sequence alignment of the transmembrane domains of all human GPCRs: phylogenetic, structural and functional implications. PLoS computational biology 12, e1004805 (2016).

13 Munk, C. et al. GPCRdb: the G protein-coupled receptor database-an introduction. British Journal of Pharmacology 173, 2195-2207 (2016).

14 Deupi, X. \& Standfuss, J. Structural insights into agonist-induced activation of G-proteincoupled receptors. Current opinion in structural biology 21, 541-551 (2011).

15 Zhou, Q. et al. Common activation mechanism of class A GPCRs. elife 8 (2019).

16 de March, C. A. et al. Conserved residues control Activation of mammalian G protein-coupled odorant receptors. Journal of the American Chemical Society 137, 8611-8616 (2015).

17 Schönegge, A.-M. et al. Evolutionary action and structural basis of the allosteric switch controlling $\beta 2$ AR functional selectivity. Nature communications 8, 1-12 (2017).

18 de March, C. A. et al. Odorant receptor 7D4 activation dynamics. Angewandte Chemie 130, 4644-4648 (2018).

19 Sakurai, T. et al. Characterization of the $\beta$-d-glucopyranoside binding site of the human bitter taste receptor hTAS2R16. Journal of Biological Chemistry 285, 28373-28378 (2010).

20 Brockhoff, A., Behrens, M., Niv, M. Y. \& Meyerhof, W. Structural requirements of bitter taste receptor activation. Proceedings of the National Academy of Sciences 107, 11110-11115 (2010).

21 Singh, N., Pydi, S. P., Upadhyaya, J. \& Chelikani, P. Structural basis of activation of bitter taste receptor T2R1 and comparison with Class A G-protein-coupled receptors (GPCRs). Journal of Biological Chemistry 286, 36032-36041 (2011).

22 Karaman, R. et al. Probing the binding pocket of the broadly tuned human bitter taste receptor TAS2R14 by chemical modification of cognate agonists. Chemical biology \& drug design $\mathbf{8 8 , 6 6 -}$ 75 (2016). 

"bitter sugars" to their human bitter taste receptor target. Scientific reports 9, 1-16 (2019).

Sandal, M. et al. Evidence for a transient additional ligand binding site in the TAS2R46 bitter taste receptor. Journal of chemical theory and computation 11, 4439-4449 (2015).

Di Pizio, A. et al. Ligand binding modes from low resolution GPCR models and mutagenesis: chicken bitter taste receptor as a test-case. Scientific reports 7, 1-11 (2017).

Thomas, A. et al. The Bitter Taste Receptor TAS2R16 Achieves High Specificity and Accommodates Diverse Glycoside Ligands by using a Two-faced Binding Pocket. Scientific reports 7, 7753, doi:10.1038/s41598-017-07256-y (2017).

27 Biarnés, $X$. et al. Insights into the binding of Phenyltiocarbamide (PTC) agonist to its target human TAS2R38 bitter receptor. PloS one 5 (2010).

28 Prasad Pydi, S., Upadhyaya, J., Singh, N., Pal Bhullar, R. \& Chelikani, P. Recent advances in structure and function studies on human bitter taste receptors. Current Protein and Peptide Science 13, 501-508 (2012).

29 Slack, J. P. et al. Modulation of bitter taste perception by a small molecule hTAS2R antagonist. Current Biology 20, 1104-1109 (2010).

30 Wang, Y. et al. Metal ions activate the human taste receptor TAS2R7. Chemical senses 44, 339347 (2019).

31 Sievers, F. et al. Fast, scalable generation of high-quality protein multiple sequence alignments using Clustal Omega. Molecular systems biology 7 (2011).

32 Waterhouse, A. M., Procter, J. B., Martin, D. M., Clamp, M. \& Barton, G. J. Jalview Version 2a multiple sequence alignment editor and analysis workbench. Bioinformatics 25, 1189-1191 (2009).

33 Lomize, M. A., Pogozheva, I. D., Joo, H., Mosberg, H. I. \& Lomize, A. L. OPM database and PPM web server: resources for positioning of proteins in membranes. Nucleic acids research 40 , D370-D376 (2012).

34 Waterhouse, A. et al. SWISS-MODEL: homology modelling of protein structures and complexes. Nucleic acids research 46, W296-W303 (2018).

35 Fredriksson, R. \& Schiöth, H. B. The repertoire of G-protein-coupled receptors in fully sequenced genomes. Molecular pharmacology 67, 1414-1425 (2005).

36 Yang, S. et al. Crystal structure of the Frizzled 4 receptor in a ligand-free state. Nature 560, 666-670 (2018).

37 Zhang, X. et al. Crystal structure of a multi-domain human smoothened receptor in complex with a super stabilizing ligand. Nature communications 8, 1-10 (2017).

38 Tsai, C.-J. et al. Crystal structure of rhodopsin in complex with a mini-Go sheds light on the principles of G protein selectivity. Science advances 4, eaat7052 (2018).

39 Miller-Gallacher, J. L. et al. The $2.1 \AA$ resolution structure of cyanopindolol-bound $\beta 1$ adrenoceptor identifies an intramembrane $\mathrm{Na}+$ ion that stabilises the ligand-free receptor. PloS one 9 (2014).

40 Staus, D. P. et al. Allosteric nanobodies reveal the dynamic range and diverse mechanisms of G-protein-coupled receptor activation. Nature 535, 448-452 (2016).

41 Zhang, $\mathrm{H}$. et al. Structure of the angiotensin receptor revealed by serial femtosecond crystallography. Cell 161, 833-844 (2015).

42 Wu, B. et al. Structures of the CXCR4 chemokine GPCR with small-molecule and cyclic peptide antagonists. Science 330, 1066-1071 (2010).

43 Peng, Y. et al. 5-HT2C receptor structures reveal the structural basis of GPCR polypharmacology. Cell 172, 719-730. e714 (2018).

44 Webb, B. \& Sali, A. Comparative Protein Structure Modeling Using MODELLER. Current Protocols in Bioinformatics 54, 5.6.1-5.6.37, doi:https://doi.org/10.1002/cpbi.3 (2016).

45 Sandal, M. et al. GOMoDo: a GPCRs online modeling and docking webserver. PloS one 8, e74092 (2013). 
Gowers, R. J. et al. MDAnalysis: a Python package for the rapid analysis of molecular dynamics simulations. Report No. 2575-9752, (Los Alamos National Lab.(LANL), Los Alamos, NM (United States), 2019).

47 Virtanen, P. et al. SciPy 1.0: fundamental algorithms for scientific computing in Python. Nature methods 17, 261-272 (2020).

48 Harris, C. R. et al. Array programming with NumPy. Nature 585, 357-362 (2020).

49 Mannige, R. V., Kundu, J. \& Whitelam, S. The Ramachandran number: an order parameter for protein geometry. PloS one 11, e0160023 (2016).

50 Studer, G., Biasini, M. \& Schwede, T. Assessing the local structural quality of transmembrane protein models using statistical potentials (QMEANBrane). Bioinformatics 30, i505-i511 (2014).

51 Ueda, T., Ugawa, S., Yamamura, H., Imaizumi, Y. \& Shimada, S. Functional interaction between T2R taste receptors and G-protein $\alpha$ subunits expressed in taste receptor cells. Journal of Neuroscience 23, 7376-7380 (2003).

52 Charlier, L. et al. How broadly tuned olfactory receptors equally recognize their agonists. Human OR1G1 as a test case. Cellular and molecular life sciences 69, 4205-4213 (2012).

53 Bushdid, C. et al. Mammalian class I odorant receptors exhibit a conserved vestibular-binding pocket. Cellular and molecular life sciences 76, 995-1004 (2019).

54 Katritch, V. et al. Allosteric sodium in class A GPCR signaling. Trends in biochemical sciences 39, 233-244 (2014).

55 Miao, Y., Nichols, S. E., Gasper, P. M., Metzger, V. T. \& McCammon, J. A. Activation and dynamic network of the M2 muscarinic receptor. Proceedings of the National Academy of Sciences 110, 10982-10987 (2013).

56 Venkatakrishnan, A. et al. Molecular signatures of G-protein-coupled receptors. Nature 494, 185-194 (2013).

57 Dalton, J. A., Lans, I. \& Giraldo, J. Quantifying conformational changes in GPCRs: glimpse of a common functional mechanism. BMC bioinformatics 16, 1-15 (2015).

58 Levit, A., Beuming, T., Krilov, G., Sherman, W. \& Niv, M. Y. Predicting GPCR promiscuity using binding site features. Journal of chemical information and modeling 54, 184-194 (2014).

59 Di Pizio, A. \& Niv, M. Y. Promiscuity and selectivity of bitter molecules and their receptors. Bioorganic \& medicinal chemistry 23, 4082-4091 (2015).

60 Nowak, S. et al. Reengineering the ligand sensitivity of the broadly tuned human bitter taste receptor TAS2R14. Biochimica et Biophysica Acta (BBA)-General Subjects 1862, 2162-2173 (2018).

61 Born, S., Levit, A., Niv, M. Y., Meyerhof, W. \& Behrens, M. The human bitter taste receptor TAS2R10 is tailored to accommodate numerous diverse ligands. Journal of Neuroscience 33, 201-213 (2013).

$62 \mathrm{Kim}, \mathrm{D}$. et al. Biased TAS2R bronchodilators inhibit airway smooth muscle growth by downregulating phosphorylated extracellular signal-regulated kinase 1/2. American journal of respiratory cell and molecular biology 60, 532-540 (2019).

63 Flock, T. et al. Selectivity determinants of GPCR-G-protein binding. Nature 545, 317-322 (2017).

64 Pydi, S. P., Singh, N., Upadhyaya, J., Bhullar, R. P. \& Chelikani, P. The third intracellular loop plays a critical role in bitter taste receptor activation. Biochimica et Biophysica Acta (BBA)Biomembranes 1838, 231-236 (2014). 